\title{
Quantification of long non-coding RNAs using qRT-PCR: comparison of different cDNA synthesis methods and RNA stability
}

\author{
Tomasz Kolenda1,2,3, Marcel Ryś ${ }^{1,2}$, Kacper Guglas ${ }^{1,2,3}$, Anna Teresiak², Renata Bliźniak², \\ Jacek Mackiewicz ${ }^{4,5,6}$, Katarzyna Lamperska²
}

\author{
${ }^{1}$ Chair of Medical Biotechnology, Poznan University of Medical Sciences, Poznan, \\ Poland \\ ${ }^{2}$ Laboratory of Cancer Genetics, Greater Poland Cancer Centre, Poznan, Poland \\ ${ }^{3}$ Postgraduate School of Molecular Medicine, Medical University of Warsaw, Warsaw, \\ Poland \\ ${ }^{4}$ Department of Medical and Experimental Oncology, Heliodor Swiecicki Clinical \\ Hospital, Poznan University of Medical Sciences, Poznan, Poland \\ ${ }^{5}$ Department of Biology and Environmental Sciences, Poznan University of Medical \\ Sciences, Poznan, Poland \\ ${ }^{6}$ Department of Diagnostics and Cancer Immunology, Greater Poland Cancer Centre, \\ Poznan, Poland
}

Submitted: 7 March 2018; Accepted: 29 May 2018

Online publication: 30 January 2019

Arch Med Sci 2021; 17 (4): 1006-1015

DOI: https://doi.org/10.5114/aoms.2019.82639

Copyright $\odot 2019$ Termedia \& Banach

\section{Abstract}

Introduction: Long non-coding RNAs (IncRNAs), a class of regulatory RNA molecules, are over 200 nucleotides long and could be used as a new potential biomarker, but their detection methods such as qRT-PCR are still not validated, and the influence of RNA degradation on IncRNA quantification is not clear. In this study, commercially available cDNA synthesis kits were tested and the influence of RNA degradation was compared.

Material and methods: Total RNA from FaDu cells was isolated and high quality RNA and highly degraded RNA samples were used. Reverse transcription was performed using three different commercially available kits and quantifications were performed using IncRNA Primer Plate and SYBR Green I Master by LightCycler 96. qRT-PCR was performed using three different cDNA samples and results are presented as the mean $C t$ values. A $p$-value $<0.05$ was considered to be significant.

Results: Lower IncRNA Ct values $(61 / 90 ; 67.78 \%)$ after qRT-PCR quantification were observed for CDNA synthesized using random hexamer primers preceded by polyA-tailing and adaptor-anchoring steps. It was observed that 9/90 (10.00\%) IncRNAs were not detectable using different cDNA synthesis methods. For $75 / 90$ (83\%) IncRNAs, RNA degradation weakly influenced IncRNA Ct values and no differences were observed between high quality RNA and degraded samples. Seventy percent of examined IncRNAs showed significantly different $C t$ values depending on RNA degradation.

Conclusions: cDNA synthesis kits with random hexamer primers preceded by polyA-tailing and adaptor-anchoring steps allows enhancement of IncRNA quantification specificity and sensitivity. In most cases degradation of RNA samples does not affect IncRNA quantification because these molecules have good stability.

Key words: IncRNA, cDNA synthesis, qRT-PCR, RNA stability and degradation.

\author{
Corresponding author: \\ Tomasz Kolenda \\ Laboratory of \\ Cancer Genetics \\ Greater Poland \\ Cancer Centre \\ 15 Garbary St, room 5025 \\ 61-866 Poznan, Poland \\ E-mail: kolenda.tomek@ \\ gmail.com
}




\section{Introduction}

The idea of using RNA as a biomarker is not new $[1,2]$. Long non-coding RNAs (IncRNAs) are molecules longer than 200 nucleotides. They are actively transcribed and do not encode any type of proteins. Knowledge about IncRNAs is still limited and thus this is an important area for further research. IncRNA molecules possess many functional domains such as RNA or DNA binding sites and protein binding sites. They may also undergo a conformational switch. Because of these domains, IncRNAs may have crucial physiological functions, such as controlling transcription, post-transcription processes, or translation, or modeling epigenetic modifications. IncRNAs participate in cellular processes such as proliferation, apoptosis, response to stress, and regulation of cell metabolism or phenotype. Disturbances in IncRNA expression are associated with cancer processes, and a growing number of studies have focused on IncRNAs as new biomarkers [3].

Detection of expression of IncRNA originating from tissue, urine, peripheral blood, serum, saliva, or urine samples can be easily performed using molecular biology methods [4-9]. It was also reported that IncRNAs can be extracted from exosomes, which are thought to be mediators in cellcell communication [10]. However, not all IncRNAs are present in each type of biological material. For example, Tang et al. observed that HOTAIR, HULC, MALAT1, MEG-3, NEAT-1, and UCA1 were present in cancer and adjacent noncancerous samples from OSCC (oral squamous cell carcinoma) patients, but only HOTAIR and MALAT1 from this group of IncRNAs were detected in some saliva samples [8].

There are many available methods to study IncRNAs: i) IncRNA immunoprecipitation; ii) InCRNA in situ hybridization; iii) Au-NP assay (gold nanoparticle-based); iv) IncRNA northern blot analysis; v) estimation of methylation status using HRM analysis (high resolution melting); vi) microarray or RNA sequencing; and vii) qRT-PCR or the newly developed ddPCR (droplet digital PCR) $[5,6,11-13]$. The most frequently used method in IncRNA studies is a hybridization assay, especially the qRT-PCR method based on SYBR-green dye and TaqMan probes [14]. The qRT-PCR experiments can be laboratory-specific. Various research groups use different primers, reference genes (or set of genes) and amplification strategies, which sometimes makes it impossible to compare the results $[15,16]$. It seems that only commercial qRT-PCR platforms could help to solve this problem. The commercially available qRT-PCR IncRNA platforms, such as the LncProfiler qPCR Array Kit (SBI), provide the possibility of simple and quick quantification of 90 IncRNAs in a single run, based on Ct (threshold cycle) analysis. In contrast to
qRT-PCR, the microarray method is well-validated technology and is more comparable among experiments, but it is more expensive [17]. Moreover, using a commercial IncRNA microarray platform, expression of over 30,000 IncRNAs can be evaluated without using the sophisticated bioinformatics methods required for NGS (next generation sequencing) data extraction [18, 19]. However, in qRT-PCR and microarray methods, detection is limited to only known IncRNA transcripts.

As mentioned above, one of the most popular methods used in IncRNA research is qRT-PCR, but this method is not standardized and different approaches to prepare cDNA are used. Moreover, the influence of RNA degradation on quantification of IncRNA is not clear. These problems should be solved before using IncRNAs as a new class of biomarker in clinical practice.

In this study, different cDNA synthesis kits were evaluated and the quality of RNA samples was compared. Kits were based on the following: i) random hexamer primers preceded by polyAtailing and adaptor-anchoring steps; ii) simple reaction using a blend of random hexamer primers and oligo(dT); iii) only random hexamer primers; and iv) only oligo(dT) (Figures 1 A-D).

\section{Material and methods}

The FaDu cell line, which is a model of hypopharynx squamous cell carcinoma, was used. The FaDu cell line was maintained in DMEM high glucose (4.5 g/l) medium (Sigma-Aldrich) supplemented with $8.85 \%(\mathrm{v} / \mathrm{v})$ fetal bovine serum (Sigma-Aldrich), $1.77 \mathrm{mM}$ L-glutamine (PAA), 0.885\% (v/v) MEM non-essential amino acid solution (PAA), $0.885 \%(\mathrm{v} / \mathrm{v})$ penicillin-streptomycin (PAA), and 8.85 mM HEPES (Sigma-Aldrich). Mycoplasma detection tests were performed routinely using the VenorGeM Mycoplasma PCR Detection Kit (Minerva Biolabs).

\section{RNA isolation, quantification, and degradation protocol}

Total RNA from FaDu cells was isolated using a High Pure miRNA isolation kit (Roche), according to the isolation protocol for total RNA (including the IncRNA fraction) from tissue and cell line samples. Quality and quantity of RNA samples were assessed using a NanoDrop spectrophotometer (Thermo Scientific), followed by $28 \mathrm{~S}$ and $18 \mathrm{~S}$ rRNA band estimation (native 1\% agarose gel electrophoresis in TAE buffer).

One high quality RNA sample was aliquoted ( $1 \mu \mathrm{g}$ of total RNA) and used for the reverse transcription reaction using three different cDNA synthesis kits. The best cDNA kit was then chosen for further experiments. 
A

PolyA-tailing
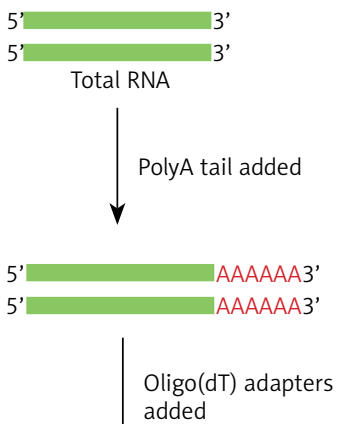
added

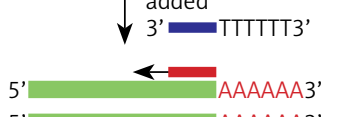

5'

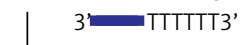

B

Hexamers and Oligo(dT)

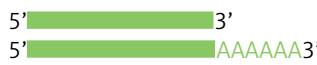

Total RNA
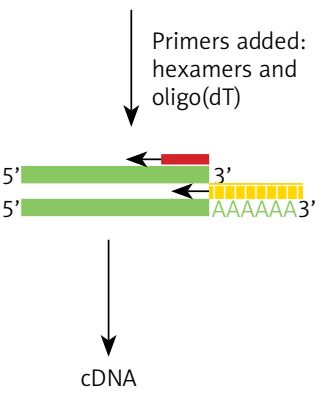

CDNA
C

Hexamers

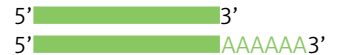

Total RNA
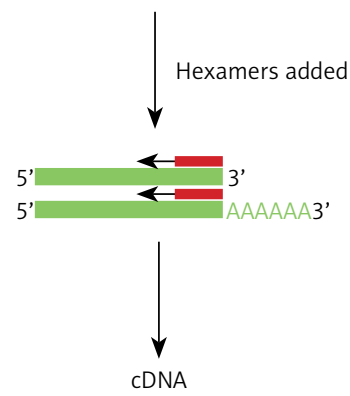

D

Oligo(dT)

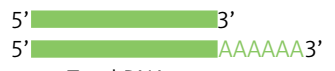

Total RNA
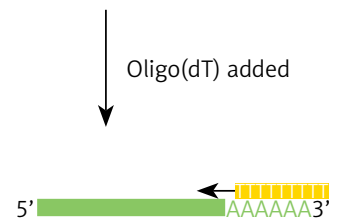

43,
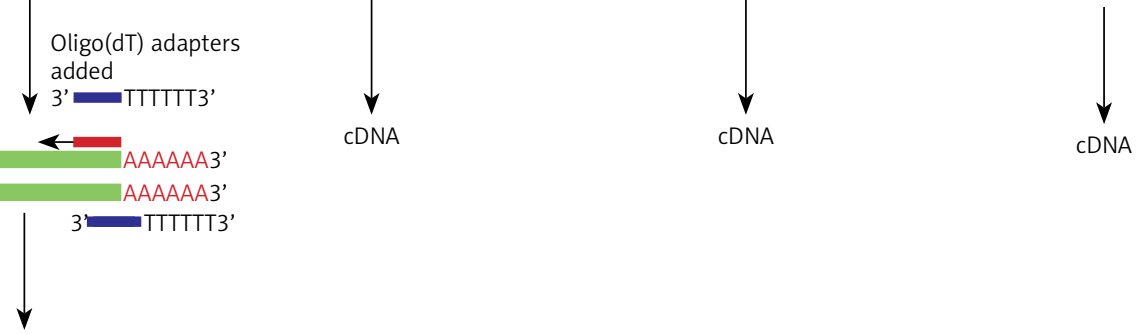

CDNA

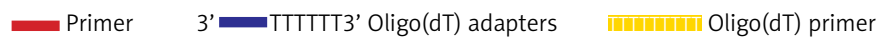

Figure 1. Comparison of different reverse transcription kits used to quantify IncRNA employing qRT-PCR methods. A - cDNA synthesis using random hexamer primers preceded by polyA-tailing and adaptor-anchoring steps, $\mathbf{B}$ - simple reaction using blend of random hexamer primers and oligo(dT), C - reaction based on random hexamer primers, D - based only on oligo(dT)

To test the influence of RNA integrity on IncRNA amplification, an RNA degradation protocol was developed. Aliquoted RNA (1 $\mu \mathrm{g} / \mathrm{tube})$ was incubated for $0,3,6,8$, and 10 days at room temperature. At all time points, the RNA quality was tested using $28 \mathrm{~S}$ and $18 \mathrm{~S}$ rRNA band estimation (native $1 \%$ agarose gel electrophoresis in TAE buffer). Additional RNA samples on day 0 and day 10 were tested using a NanoDrop spectrophotometer.

High quality (visible $28 \mathrm{~S}$ and $18 \mathrm{~S}$ rRNA bands), degraded (lack of 28S rRNA band and visible $18 \mathrm{~S}$ rRNA band), and highly degraded RNA samples (lack of a $28 \mathrm{~S}$ or $18 \mathrm{~S}$ rRNA band, visible smear of RNA) were used for CDNA synthesis and the qRT$P C R$ reaction was performed.

\section{cDNA synthesis}

Reverse transcription was performed using three different commercially available kits: i) LncProfiler qPCR Array Kit (SBI); ii) iScript cDNA Synthesis Kit (Bio-Rad); and iii) First Strand cDNA Synthesis Kit (Fermentas). For all reactions, the same amount of total RNA (1 $\mu \mathrm{g} /$ reaction) from the same isolation was used. All experiments (RNA isolation, CDNA synthesis and qRT-PCR) were performed in triplicate.

\section{LncProfiler qPCR array kit (SBI)}

Reverse transcription is based on 3 steps: i) poly-A tailing; ii) annealing anchor dT adaptor; and iii) cDNA synthesis. In the first step, $5 \mu$ l of total RNA $(1 \mu \mathrm{g})$ was mixed with $2 \mu \mathrm{l}$ of $5 \times$ PolyA Buffer, $1 \mu \mathrm{l}$ of $\mathrm{MnCl}_{2}, 1.5 \mu \mathrm{l}$ of ATP, and $0.5 \mu \mathrm{l}$ of PolyA Polymerase and incubated for $30 \mathrm{~min}$ at $37^{\circ} \mathrm{C}$. In the next step, $0.5 \mu$ of Oligo(dT) Adapter was added. The reaction was heated for $5 \mathrm{~min}$ at $60^{\circ} \mathrm{C}$ and then cooled to room temperature for $2 \mathrm{~min}$. In the third step, $4 \mu \mathrm{l}$ of RT Buffer, $2 \mu$ of dNTP mix, $1.5 \mu \mathrm{l}$ of $0.1 \mathrm{M}$ DTT, $1.5 \mu \mathrm{l}$ of random Primer Mix, and $1 \mu \mathrm{l}$ of Reverse transcriptase were added and incubated for $60 \mathrm{~min}$ at $42^{\circ} \mathrm{C}$ followed by heating for $10 \mathrm{~min}$ at $95^{\circ} \mathrm{C}$.

\section{iScript cDNA synthesis Kit (Bio-Rad)}

The following were mixed: $5 \mu$ of total RNA $(1 \mu \mathrm{g}), 4 \mu \mathrm{l}$ of $5 \times$ iScript Reaction Mix (contained blend of oligo(dT) and random hexamer primers), $1 \mu \mathrm{l}$ of iScript Reverse Transcriptase, and nuclease free water (up to $20 \mu \mathrm{l}$ ). The reaction was incubated for $5 \mathrm{~min}$ at $25^{\circ} \mathrm{C}$ and followed by $20 \mathrm{~min}$ at $46^{\circ} \mathrm{C}$ and $1 \mathrm{~min}$ at $95^{\circ} \mathrm{C}$.

\section{First strand cDNA synthesis Kit (Fermentas)}

Two specific mixes of primers were used: the oligo(dT) primer and the random hexamer primer mixes. The reaction mixture contained $1 \mu \mathrm{g}$ total RNA, and oligo(dT) or random hexamer primers $(1 \mu \mathrm{l})$, and it was filled up to a volume of $11 \mu \mathrm{l}$ using nuclease free water. The reactions were incubated at $65^{\circ} \mathrm{C}$ for $5 \mathrm{~min}$, followed by cooling on ice and 
collecting drops by brief centrifugation. Next, $4 \mu \mathrm{l}$ of $5 \times$ reaction buffer, $1 \mu$ l of RiboLock Ribonuclease Inhibitor, $2 \mu \mathrm{l}$ of $10 \mathrm{mM}$ dNTP mix, and $2 \mu \mathrm{l}$ of M-MuLV reverse transcriptase were added. Reactions were incubated at $37^{\circ} \mathrm{C}$ for $60 \mathrm{~min}$ followed by $10 \mathrm{~min}$ at $70^{\circ} \mathrm{C}$ (for reactions with hexamer primers, the incubation procedure was followed by a sequential incubation step of $10 \mathrm{~min}$ at $25^{\circ} \mathrm{C}$ ).

\section{qRT-PCR reaction}

CDNA was mixed with $1.750 \mathrm{ml}$ of $2 \times$ LightCycler 480 SYBR Green I Master buffer (Roche) and $1.480 \mathrm{ml}$ of nuclease free water, and $26 \mu \mathrm{l}$ of the mixture was dropped into wells on a 96-well qRT-PCR plate. Next, $4 \mu \mathrm{l}$ of IncRNA primers from Primer Plate (component of the LncProfiler qPCR Array Kit) was loaded onto the plate and the $\mathrm{PPCR}$ reaction was performed using the following protocol: preincubations $\left(50^{\circ} \mathrm{C}\right.$ for $2 \mathrm{~min}$ and $95^{\circ} \mathrm{C}$ for $10 \mathrm{~min}), 60$ cycles of 2-step amplification $\left(95^{\circ} \mathrm{C}\right.$ for $15 \mathrm{~s}$ and $60^{\circ} \mathrm{C}$ for $1 \mathrm{~min}$ ), and a melting step. Reactions were performed using a LightCycler 96 (Roche).

\section{Statistical analysis}

To compare $C t$ values, the statistical analysis was performed using GraphPad Prism 5 software with an unpaired $t$-test or a one-way analysis of variance (ANOVA) followed by Tukey's post-hoc test. All qRT-PCR experiments were performed three times using three different cDNA samples and are presented as the mean $C t$ values. Error bars represent the standard deviation (SD) and a $p$-value $<0.05$ was considered to be significant.

\section{Results}

In this study, different cDNA synthesis kits based on: i) random hexamer primers preceded by polyA-tailing and adaptor-anchoring steps; ii) simple reaction using a blend of random hexamer primers and oligo(dT); iii) using only random hexamer primers; and iv) only oligo(dT) were tested. We assumed that the lower the $C t$ value was, the more sensitive and precise was the method of CDNA synthesis. Comparison of mean qRT-PCR Ct values is presented on a heat map (Figure $2 \mathrm{~A}$ ). Lower Ct values were observed for 61/90 (67.78\%) of examined IncRNAs where cDNA was synthesized using random hexamer primers preceded by polyA-tailing and adaptor-anchoring steps. However, in the case of $15 / 90$ (16.67\%) IncRNAs, higher $C t$ values were observed for other methods (Figures $2 \mathrm{~A}$ and $\mathrm{B}$ ).

Heat map and clustering showed that qRTPCR Ct values for cDNA obtained using random hexamer primers preceded by polyA-tailing and adaptor-anchoring steps are distinct from other examined cDNA synthesis kits, which are similar to each other and clustered together (Figure $2 \mathrm{~A}$ ). Moreover, 9/90 (10\%) of the examined IncRNAs were detected only in the cDNA samples obtained by random hexamer primers preceded by polyA-tailing and adaptor-anchoring steps. However, for 5/90 (5.56\%) IncRNAs, no IncRNA amplification was observed, and these IncRNAs were detected in cDNA samples synthesized using a combination of random hexamer primers and oligo(dT), and with only random hexamer primers or only oligo(dT) (Figures $2 \mathrm{~A}$ and $\mathrm{B}$ ).

For the most frequently described IncRNAs in cancers and in head and neck squamous cell carcinomas in particular [3], significant changes were observed among qRT-PCR Ct values obtained using different cDNA synthesis kits. For H19 $(p<0.0001)$, HOTTIP $(p=0.0011)$, the GAS5 family $(p<0.0001)$, the MEG3 family $(p=0.0001)$, Air $(p<0.0001)$, and HOTAIRM1 $(p<0.0001)$, lower $C t$ values were observed (32.55 \pm 4.12 vs. 42.49 $\pm 3.52)$, while for ANRIL $(p=0.0045)$ and HOTAIR $(p=0.0053)$, higher $C t$ values (34.18 \pm 0.33 vs. $31.68 \pm 1.46$ ) were observed for cDNA obtained with random hexamer primers preceded by polyA-tailing and adaptor-anchoring steps compared to the rest of the tested kits (Figure $2 \mathrm{C}$ ).

The influence of total RNA degradation on qRT$P C R$ quantification was then investigated. Freshly isolated RNA was aliquoted ( $V=5 \mu \mathrm{l} /$ sample, $C=$ $1 \mu \mathrm{g} /$ sample) and incubated at room temperature for $3,6,8$, and 10 days. Degradation of total RNA was observed (Figure $3 \mathrm{~A}$ ). After 3 days of incubation, no $28 \mathrm{~S}$ band but a smear of the 18S rRNA band was observed until day 10, when RNA was highly degraded (no $28 \mathrm{~S}$ or $18 \mathrm{~S}$ rRNA bands; Figure $3 \mathrm{~A}$ ). The degradation process caused changes in RNA sample absorbance after 10 days of incubation that was measured using a NanoDrop spectrophotometer. The UV absorbance increased and is observed as an effect of apparent concentration change from $206.3 \mathrm{ng} / \mu \mathrm{l}$ measured immediately after isolation to $235.27 \mathrm{ng} / \mu \mathrm{l}$ measured after 10 days of incubation ( $p=0.0227$; Figure $3 \mathrm{~B}$ ).

cDNA synthesis using random hexamer primers preceded by polyA-tailing and adaptor-anchoring steps and qRT-PCR using the LncProfiler qPCR Array kit were performed for RNA samples from the day of isolation as well as after incubation for 3 and 10 days. For $83 \%$ (75/90) of IncRNAs, there were no significant differences $(p>0.05)$ in Ct values between high quality RNA and degraded RNA (day 3 or 10). For IncRNAs: Jpx, PSF inhibiting RNA, PTENP1, SAF, SNHG5, and the mRNA GAPDH there were no differences between $C t$ values of cDNA synthesized using RNA samples after isolation or after incubation for 3 days, but significantly higher Ct values were observed for the samples when the qRT-PCR was performed based on RNA incu- 


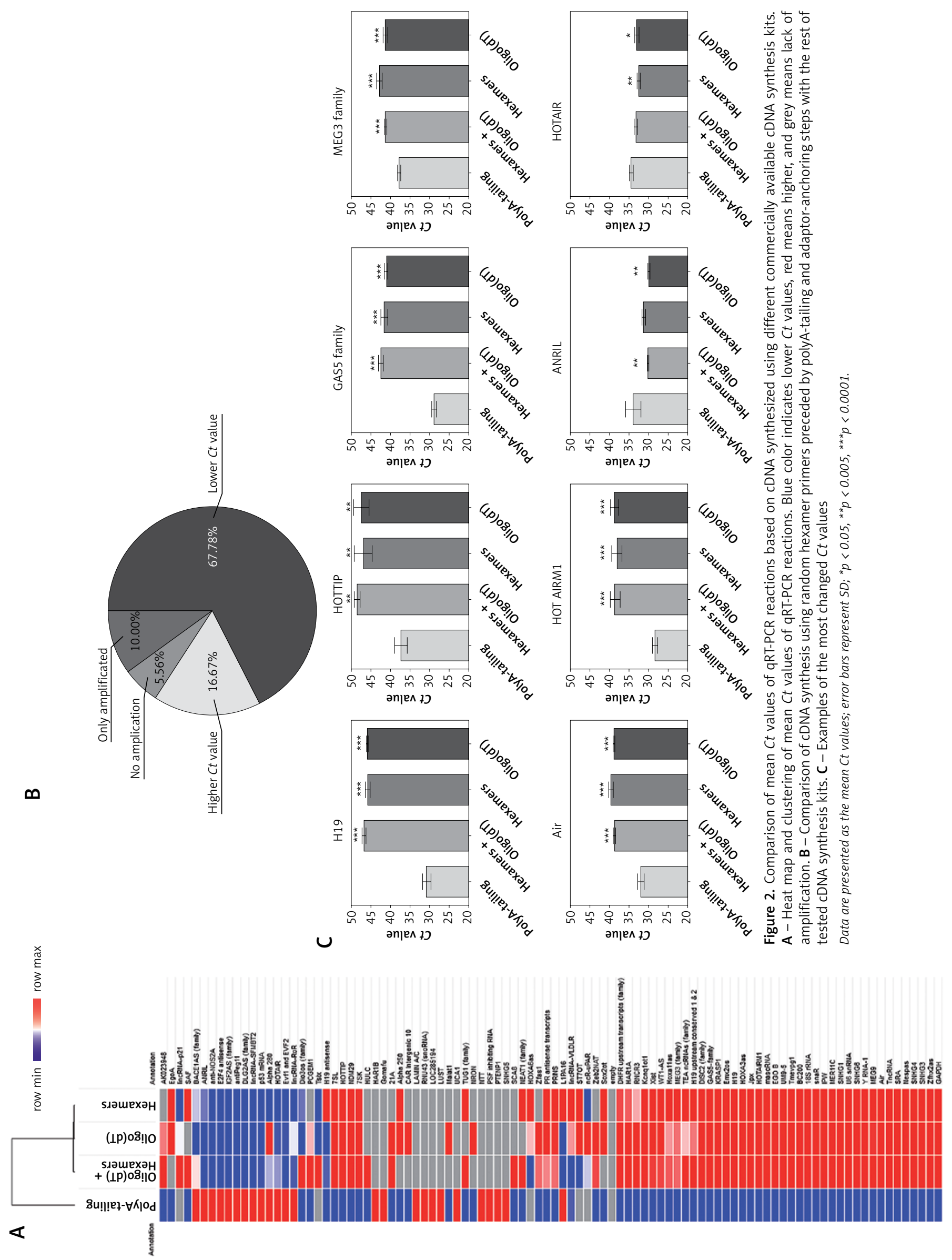


A

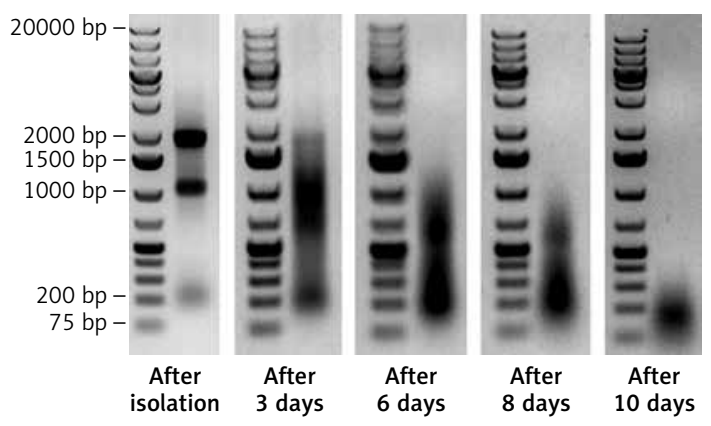

B

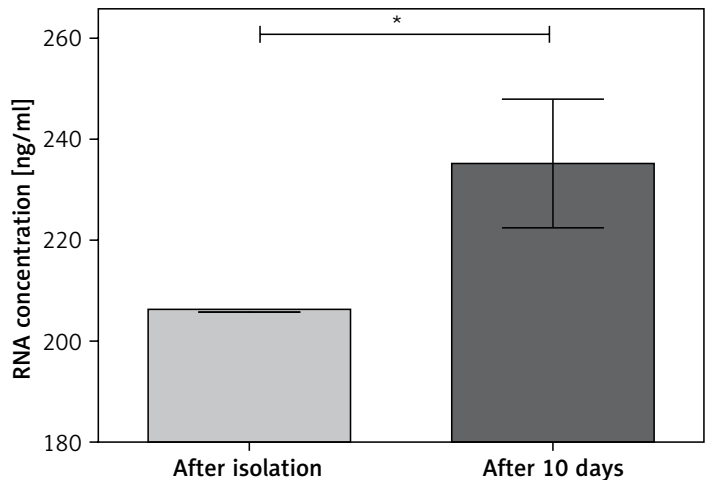

Figure 3. Degradation of RNA used for cDNA synthesis. A - Native electrophoresis in $1 \%$ agarose gel of RNA samples at different time points. Marker GeneRuler $1 \mathrm{~kb}$ Plus DNA Ladder (Thermo Scientific). B - Mean RNA concentration measured immediately after isolation and after 10 days of incubation at room temperature

Data are presented as the mean Ct values; error bars represent $S D ;{ }^{*} p<0.05$.

bated for 10 days (34.92 \pm 0.28 vs. $37.71 \pm 1.17$, $p=0.0225 ; 36.51 \pm 0.05$ vs. $38.78 \pm 0.40, p=$ $0.0035 ; 37.72 \pm 0.72$ vs. $41.28 \pm 1.75, p=0.0221$; $32.90 \pm 0.21$ vs. $35.01 \pm 0.87, p=0.0291 ; 29.55$ \pm 0.58 vs. $33.32 \pm 0.58, p=0.0018$, and 25.10 \pm 1.10 vs. $27.76 \pm 0.81, p=0.0150$, respectively). Moreover, these differences were also observed between samples incubated for 3 and 10 days ( $35.25 \pm 0.62$ vs. $37.71 \pm 1.17 ; 36.98 \pm 0.54$ vs. 38.78 $\pm 0.40 ; 37.48 \pm 0.50$ vs. $41.28 \pm 1.75 ; 32.82 \pm 0.53$ vs. $35.01 \pm 0.87 ; 30.15 \pm 0.67$ vs. $33.32 \pm 0.58$, and $25.05 \pm 0.53$ vs. $27.76 \pm 0.81$, respectively). For the IncRNAs Tmevpg1 and Xist higher $C t$ values were noted only between days 3 and 10 (32.52 \pm 0.52 vs. $33.77 \pm 0.41, p=0.0386$, and $35.34 \pm 0.35$ vs. 36.97 $\pm 0.82, p=0.0306$, respectively) and for Zfas 1 after isolation and after incubation for 10 days (28.71 \pm 0.70 vs. $31.76 \pm 1.42, p=0.0406)$. qRT-PCR performed using RNA incubated for 10 days provided better results (lower Ct values) compared to cDNA based on RNA after isolation in the case of NEAT1 (family), Nespas, NRON and ST7OT (27.45 \pm 0.50 vs. $28.91 \pm 0.02, p=0.0490 ; 30.97 \pm 1.18$ vs. 34.36 $\pm 0.66, p=0.0217 ; 32.87 \pm 0.36$ vs. $34.01 \pm 0.13$, $p=0.0263$, and $28.04 \pm 0.41$ vs. $29.74 \pm 0.36$, $p=0.0214$, respectively), and for lincRNA-RoR compared to cDNA based on RNA incubated for 3 days (34.27 \pm 0.31 vs. $35.36 \pm 0.23, p=0.0238$ ). For the IncRNAs MER11C and NTT lower Ct values were observed for RNA incubated for 3 days as well as for 10 days compared to RNA after isolation (30.51 \pm 0.48 and $30.31 \pm 0.60$ vs. $32.15 \pm 0.01$, $p=0.0257$, and $32.90 \pm 0.49$ and $32.72 \pm 0.45$ vs. $34.97 \pm 0.23, p=0.0047$, respectively; Figure 4).

\section{Discussion}

Similarly to most researchers, we also chose the qRT-PCR method to study IncRNA expression because of its simplicity and quick sample analysis [20]. However, the main issue for qRT-PCR is selection of the proper methodology in the experimental workflow, but there are no experimental data comparing various approaches to this issue. In this study, we compared three different commercially available cDNA synthesis kits. Based on total RNA (including the IncRNA fraction) we obtained the CDNA, and this could be used to quantify expression of IncRNA using the commercially available LncProfiler qPCR Array Kit (SBI). We assumed that the lower the $C t$ value was, the more sensitive and precise was the method. Comparing three different approaches, we observed the best results (low Ct values) for the method using cDNA synthesis by random hexamer primers preceded by polyA-tailing and adaptor-anchoring steps. Generally, results of simple reactions using a blend of random hexamer primers and oligo(dT) or just random hexamer primers or oligo(dT) alone could incorrectly suggest that there was no expression of some IncRNAs or that the expression was at a very low level. It should be noted that some of the IncRNAs possess endogenous polyA tails but others do not. Moreover, most IncRNAs are present in samples at low copy numbers, which causes difficulties for their quantification [21, 22]. These two IncRNA features require the use of cDNA kits with an additional step of adding polyA tails and annealing anchor (dT) adapters. This approach allows enhancement of the specificity and sensitivity of IncRNA quantification, which we observed. Unfortunately, in most of the studies, cDNA is prepared using kits containing a mixture of oligo(dT) and/or random hexamer primers [14, 23, 24], where oligo(dT) is not functional for IncRNA molecules without a polyA tail. It is well known that specific primers decrease background priming, but random and oligo(dT) primers maximize the number of mRNA molecules that can be analyzed from a small sample of RNA [25]. We observed that artificially adding polyA tails and then 

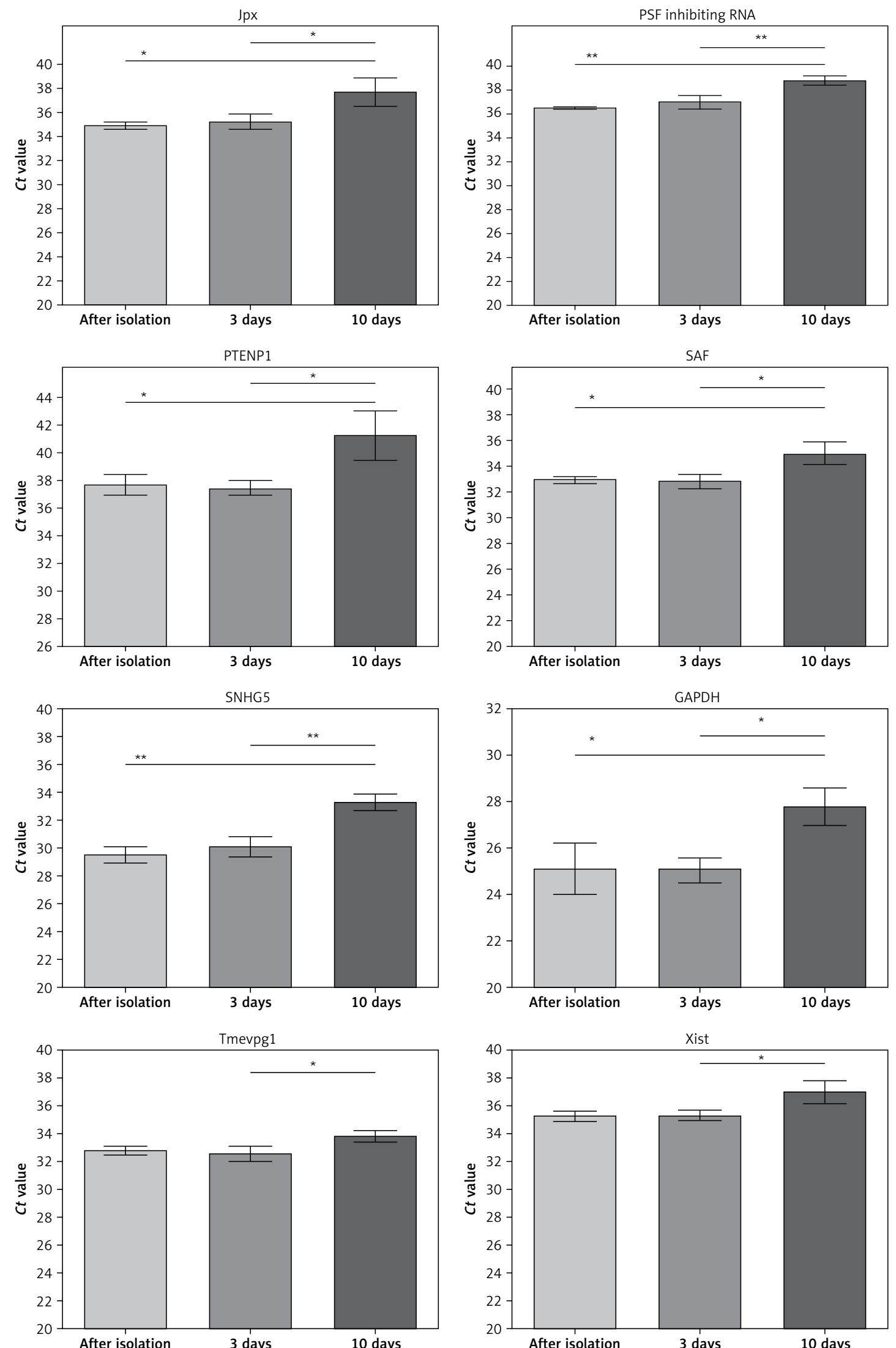

Figure 4. Comparison of mean Ct values of changed IncRNAs and GAPDH mRNA amplified with cDNA synthesis using random hexamer primers preceded by polyA-tailing and adaptor-anchoring steps and RNA samples just after isolation and after 3 and 10 days incubation at room temperature

Data are presented as the mean Ct values; error bars represent $S D ;{ }^{*} p<0.05,{ }^{* *} p<0.005$. 

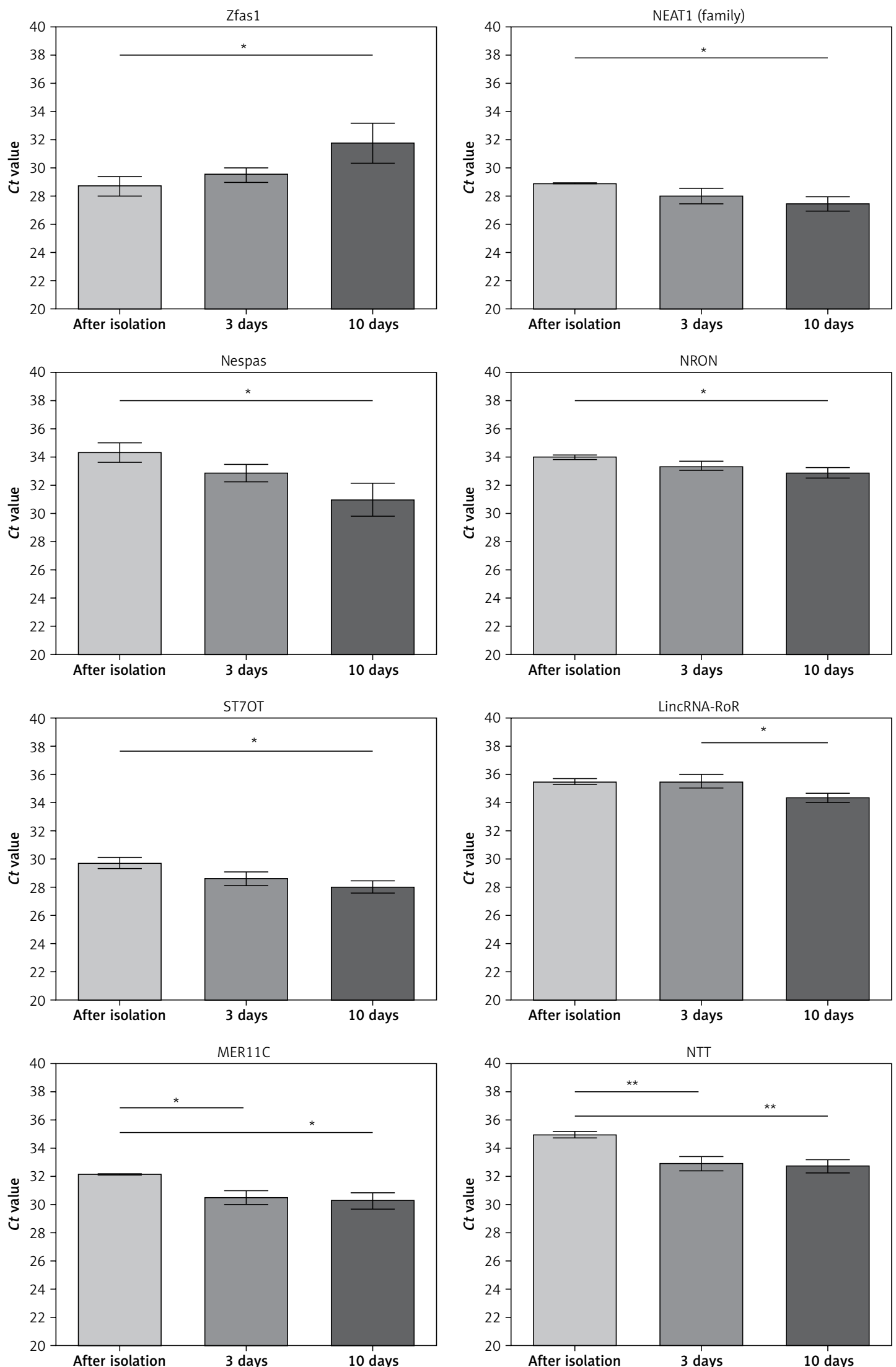

Figure 4. Cont. 
using annealing anchor (dT) adapters (analogs of oligo(dT) primers) can help to quantify IncRNAs more precisely.

One possible explanation of the difficulties in quantification of some IncRNAs observed by us is an influence of molecular structure features such as length, GC content and molecule folding. It is well known that short RNA molecules such as miRNAs are difficult to quantify by qRT-PCR and require specific cDNA synthesis kits with adapter or polyA tail addition. Longer molecules seem to be easier to quantify than short RNAs such as miRNAs. On the other hand, a lot of secondary structures such as hairpin loops are problematic for cDNA synthesis and require a higher temperature for relaxing RNA molecules, making them available for reverse transcriptase [26, 27]. Moreover, one of the kits used by us possesses the DTT (dithiothreitol) enhancer, which helps to amplify templates rich in GC sequences, and it contributed to the better qRT-PCR results [28]. Because of the structural characteristics, some IncRNAs seem to need addition of a polyA tail or a higher temperature for a longer time during CDNA synthesis. We suggest that some of the results could be overlooked because an improper cDNA synthesis approach was used and specific IncRNA was not quantified.

The next problem is the stability of IncRNAs and the influence of RNA integrity on qRT-PCR quantification. Because of the length of IncRNAs (more than $200 \mathrm{nt}$ ), they are considered to be less stable and easier to degrade compared with short RNA molecules such as miRNAs. We analyzed the influence of RNA stability on quantification of $\ln$ cRNAs using a cDNA kit (polyA tailing with anchor (dT) adapter annealing and cDNA synthesis using random primer mix), and we compared samples of high quality total RNA (visible $28 \mathrm{~S}$ and $18 \mathrm{~S}$ rRNA bands), degraded (no $28 \mathrm{~S}$ band and visible smear of $18 \mathrm{~S}$ rRNA band) and highly degraded RNA (no $28 \mathrm{~S}$ or $18 \mathrm{~S}$ rRNA bands). Degradation causes changes in RNA structure increasing the amount of short RNA molecules, which strongly affects RNA detection $[29,30]$. Our results indicated that most of the examined IncRNAs are stable, even when the total RNA is highly degraded. Our observations are supported by the results obtained by Kraus et al. They reported that some IncRNAs are more stable than miRNAs that are about 22 nucleotides long [31, 32].

However, some long transcripts, such as mRNAs, are sensitive to degradation, and in this case, RNA integrity is an important factor affecting qRT-PCR quantification. It is postulated that the IncRNA half-life depends on its coding place in the genome, on posttranscriptional modifications, and on subcellular localization and its function [33]. Some authors have noted that localization of In-
cRNAs in the genome may influence their transcript stability, especially intragenic and cis-antisense IncRNAs compared with those derived from introns (half-life more than $16 \mathrm{~h}$ ) [8, 33]. We noted that some IncRNAs are stable for much longer. After 3 days, we did not observe changes in Ct values in most of the examined IncRNAs, while after 10 days, changes in $C t$ values were observed only in some IncRNAs. Detection of some IncRNAs in saliva confirms the high stability of these molecules [8]. Moreover, IncRNAs isolated from the plasma are resistant to RNase $A$ digestion and overnight incubation at room temperature [9]. Generally, low RNA stability may cause difficulties in analyzing some long coding or non-coding RNA transcripts obtained from archived formalin-fixed paraffin-embedded (FFPE) blocks [34]. This problem is solved by applying the simple modification of measuring the IncRNA expression level by reaction with three different pairs of non-overlapping primers [35]. However, RNA is both degraded and modified under the archiving process in FFPE blocks, which makes this RNA more difficult to analyze [36].

It is well known that the main features of an ideal biomarker molecule are simplicity of acquisition, diversity of sources where it occurs, and ease of measurement methodology [20, 37]. We postulate that under appropriate processing conditions, IncRNA seems to meet the characteristics of biomarker molecules. However, standardization procedures should be applied before clinical use of IncRNA biomarkers.

In conclusion, we recommend the following: i) use of cDNA kits designed for IncRNA or CDNA synthesis preceded by polyA tailing with anchor (dT) adapter annealing; ii) use of highly thermostable reverse transcriptase designed for long reactions at higher temperatures; and iii) use of high RNA quality is generally recommended, but most IncRNAs seem to be stable and can be quantified in degraded RNA samples.

\section{Acknowledgments}

Marcel Ryś and Kacper Guglas contributed equally to this work.

This work was supported by Greater Poland Cancer Centre - grant no. 21/2015 (113) and grant no. 13/2016 (128), and by the National Science Centre, Poland, allocated on the basis of decision no. 2016/21/B/NZ7/01773.

The language correction and text formatting were performed by American Manuscript Editors (americanmanuscripteditors.com).

\section{Conflict of interest}

The authors declare no conflict of interest. 


\section{References}

1. Lamperska KM, Kozlowski P, Kolenda T, et al. Unpredictable changes of selected miRNA in expression profile of HNSCC. Cancer Biomark 2016; 16: 55-64.

2. Kolenda T, Przybyła W, Teresiak A, Mackiewicz A, Lamperska KM. The mystery of let-7d - a small RNA with great power. Contemp Oncol 2014; 18: 293-301.

3. Kolenda T, Guglas K, Ryś M, et al. Biological role of IncRNA in head and neck cancers. Rep Pract Oncol Radiother 2017; 22: 378-88.

4. Yarmishyn AA, Kurochkin IV. Long noncoding RNAs: a potential novel class of cancer biomarkers. Front Genet 2015; 6: 145 .

5. Eissa S, Matboli M, Essawy NO, Shehta M, Kotb YM. Rapid detection of urinary long non-coding RNA urothelial carcinoma associated one using a PCR-free nanoparticle-based assay. Biomarkers 2015; 20: 212-7.

6. Zhang H, Zhao L, Wang YX, Xi M, Liu SL, Luo LL. Long non-coding RNA HOTTIP is correlated with progression and prognosis in tongue squamous cell carcinoma. Tumour Biol 2015; 36: 8805-9.

7. Fayda M, Isin M, Tambas M, et al. Do circulating long non-coding RNAs (IncRNAs) (LincRNA-p21, GAS 5, HOTAIR) predict the treatment response in patients with head and neck cancer treated with chemoradiotherapy? Tumour Biol 2016; 37: 3969-78.

8. Tang H, Wu Z, Zhang J, Su B. Salivary IncRNA as a potential marker for oral squamous cell carcinoma diagnosis. Mol Med Rep 2013; 7: 761-6.

9. Zhou X, Yin C, Dang Y, Ye F, Zhang G. Identification of the long non-coding RNA H19 in plasma as a novel biomarker for diagnosis of gastric cancer. Sci Rep 2015; 5: 11516.

10. Gezer U, Özgür E, Cetinkaya M, Isin M, Dalay N. Long non-coding RNAs with low expression levels in cells are enriched in secreted exosomes. Cell Biol Int 2014; 38 : 1076-9.

11. Feng Y, Hu X, Zhang Y, Zhang D, Li C, Zhang L. Methods for the study of long noncoding RNA in cancer cell sig naling. Methods Mol Biol 2014; 1165: 115-43.

12. Wojdacz TK, Dobrovic A, Algar EM. Rapid detection of methylation change at $\mathrm{H} 19$ in human imprinting disorders using methylation-sensitive high-resolution melting. Hum Mutat 2008; 29: 1255-60.

13. Dodd DW, Gagnon KT, Corey DR. Digital quantitation of potential therapeutic target RNAs. Nucleic Acid Ther 2013; 23: 188-94.

14. Shi T, Gao G, Cao Y. Long noncoding RNAs as novel biomarkers have a promising future in cancer diagnostics. Dis Markers 2016; 2016: 9085195.

15. Wong ML, Medrano JF. Real-time PCR for mRNA quantitation. Biotechniques 2005; 39: 75-85.

16. Bustin SA, Nolan T. Pitfalls of quantitative real-time reverse-transcription polymerase chain reaction. J Biomol Tech 2004; 15: 155-66.

17. Gee HE, Buffa FM, Camps C, et al. The small-nucleolar RNAs commonly used for microRNA normalisation correlate with tumour pathology and prognosis. Br J Cancer 2011; 104: 1168-77.

18. Oleksiewicz U, Tomczak K, Woropaj J, Markowska M, Stępniak P, Shah PK. Computational characterisation of cancer molecular profiles derived using next generation sequencing. Contemp Oncol (Pozn) 2015; 19: A78-91.

19. Tomczak K, Czerwińska P, Wiznerowicz M. The Cancer Genome Atlas (TCGA): an immeasurable source of knowledge. Contemp Oncol (Pozn) 2015; 19: A68-77.
20. Guglas K, Bogaczyńska M, Kolenda T, et al. IncRNA in HNSCC: challenges and potential. Contemp Oncol (Pozn) 2017; 21: 259-66.

21. Ravasi T, Suzuki H, Pang KC, et al. Experimental validation of the regulated expression of large numbers of non-coding RNAs from the mouse genome. Genome Res 2006; 16: 11-9.

22. Derrien T, Johnson R, Bussotti G, et al. The GENCODE v7 catalog of human long noncoding RNAs: analysis of their gene structure, evolution, and expression. Genome Res 2012; 22: 1775-89.

23. Fang Z, Wu L, Wang L, Yang Y, Meng Y, Yang H. Increased expression of the long non-coding RNA UCA1 in tongue squamous cell carcinomas: a possible correlation with cancer metastasis. Oral Surg Oral Med Oral Pathol Oral Radiol 2014; 117: 89-95.

24. Yang YT, Wang YF, Lai JY, et al. Long non-coding RNA UCA1 contributes to the progression of oral squamous cell carcinoma by regulating the WNT/beta-catenin signaling pathway. Cancer Sci 2016; 107: 1581-9.

25. Malewski T, Malewska A, Rutkowski R. RT-PCR technique and its applications. State-of the-art. J Anim Feed Sci 2003; 12: 403-16.

26. Harrison GP, Mayo MS, Hunter E, Lever AM. Pausing of reverse transcriptase on retroviral RNA templates is influenced by secondary structures both 5' and 3' of the catalytic site. Nucleic Acids Res 1998; 26: 3433-42.

27. Brooks EM, Sheflin LG, Spaulding SW. Secondary structure in the 3' UTR of EGF and the choice of reverse transcriptases affect the detection of message diversity by RT-PCR. Biotechniques 1995; 19: 806-12, 814-5.

28. Green MR, Sambrook J. Molecular Cloning: A Laboratory Manual (Fourth Edition). Vol. 1. Cold Spring Harbor Laboratory Press, New York 2012; 484-9.

29. Vermeulen J, De Preter K, Lefever S, et al. Measurable impact of RNA quality on gene expression results from quantitative PCR. Nucleic Acids Res 2011; 39: e63.

30. Fleige S, Pfaffl MW. RNA integrity and the effect on the real-time qRT-PCR performance. Mol Aspects Med 2006; 27: $126-39$.

31. Kraus TF, Greiner A, Guibourt V, Lisec K, Kretzschmar HA. Identification of stably expressed IncRNAs as valid endogenous controls for profiling of human glioma. J Cancer 2015; 6: 111-9.

32. Kraus TF, Greiner A, Guibourt V, Kretzschmar HA. Long non-coding RNA normalisers in human brain tissue. J Neural Transm (Vienna) 2015; 122: 1045-54.

33. Clark MB, Johnston RL, Inostroza-Ponta M, et al. Genome-wide analysis of long noncoding RNA stability. Genome Res 2012; 22: 885-98.

34. Kokkat TJ, Patel MS, McGarvey D, LiVolsi VA, Baloch ZW. Archived formalin-fixed paraffin-embedded (FFPE) blocks: a valuable underexploited resource for extraction of DNA, RNA, and protein. Biopreserv Biobank 2013; 11: 101-6.

35. Kong H, Zhu M, Cui F, et al. Quantitative assessment of short amplicons in FFPE-derived long-chain RNA. Sci Rep 2014; 4: 7246.

36. Hamatani K, Eguchi H, Takahashi K, et al. Improved RTPCR amplification for molecular analyses with long-term preserved formalin-fixed, paraffin-embedded tissue specimens. J Histochem Cytochem 2006; 54: 773-80.

37. Kolenda T, Teresiak A, Kapałczyńska M, et al. Let-7d and miR-18a as biomarkers of head and neck cancers. Zeszyty Naukowe WCO, Letters in Oncology Science 2015; 12: 37-47. 